\title{
Double photoionization: recent results at Daresbury and perspectives at Trieste
}

\author{
L. AVALDI, G. DAWBER ${ }^{*}$, R.I. HALL ${ }^{* *}$, G.C. KING ${ }^{*}$, M.A. MacDONALD*** and A. McCONKEY*
}

IMAI del CNR, Area della Ricerca di Roma, Italy

${ }^{*}$ Department of Physics, Manchester University, Manchester, U.K.

** Laboratoire de Dynamique Atomique et Moléculaire, CNRS and Université P. et M. Curie, Paris, France

*** SERC Daresbury Laboratory, Daresbury, Warrington, U.K.

\section{ABSTRACT}

Recent experimental investigations of double ionization of rare gases and diatomic molecules induced by photons from a synchrotron radiation source are presented. A novel technique where two electrons with near zero energy are detected in coincidence is described.

New experimental developments of the photoelectron-photoelectron coincidence technique, made possible by third generation synchrotron radiation sources, are addressed

\section{INTRODUCTION}

The process of one-photon double ionization in free atoms and molecules is a direct manifestation of electron-electron correlations. Since a single photon can interact only with one target electron, double ionization processes are entirely due to electron correlations, i.e. to the Coulomb interaction among the target electrons.

To achieve a complete view of the process

$$
h v+A \rightarrow A^{2+}+e_{1}\left(E_{1}\right)+e_{2}\left(E_{2}\right)
$$

several pieces of information have to be obtained experimentally (1). First of all double ionisation events have to be isolated from other possible processes. This implies a knowledge of the double ionization potentials, $\mathrm{IP}^{2+}$, and the spectroscopy of the doubly charged ions. One also needs to know the overall strength of the process, which means determining the total cross-section, $\sigma^{2+}(E)$, the energy partitioning between the two electrons $\left(d \sigma / d E_{1}\right)$, their angular distribution $\left(d^{2} \sigma / d E_{1} d \Omega_{1}\right)$, and relative angular distribution ( $d^{3} \sigma / d E_{1} d \Omega_{1} d \Omega_{2}$ ). Moreover one needs to investigate how $d^{2} \sigma / d E_{1} d \Omega_{1}$ and $d^{3} \sigma / d E_{1} d \Omega_{1} d \Omega_{2}$ change with the excess energy $E=h v-I^{2+}$.

In the case of double photoionization of molecules the process ends up with an even more complex final state

$$
\begin{array}{r}
h v+A B \rightarrow A^{2+}+e_{1}\left(E_{1}\right)+e_{2}\left(E_{2}\right) \\
y A^{+}+B^{+}
\end{array}
$$


because the molecular doubly charged ions, dications in chemistry language, either dissociate rapidly or are metastable with a local minimum in the potential curve at an energy higher than the $\mathrm{A}^{+}+\mathrm{B}^{+}$dissociation limit.

Due to the complicated fragmentation pattern of the double ionization process a full view of its dynamics can be obtained only via a variety of coincidence experiments, which enable the simultaneous detection of, at least, two of the charged products in the final states. The present technological challenge consists in the development of more and more refined multicoincidence techniques that will enable us to get as much information as possible from a single experiment.

Several coincidence techniques have been applied to study the formation and decay of doubly charged ions. They are referred by acronyms that show the types of particles detected. Thus for example PEPICO is a photoelectron-photion coincidence experiment (TPEPICO when a threshold electron is detected), while PEPECO and PIPICO indicates photoelectron-photoelectron and photoion-photoion coincidence experiments, respectively. A detailed review of all these techniques can be found in the paper by Eland (2).

This progress report deals only with PEPECO experiments. In particular the first two sections will be devoted to the study of double ionization of rare gases in the threshold region and to the spectroscopy of molecular dications by a novel technique which joins the selectivity of the coincidence measurements to the high sensitivity and resolution of threshold spectroscopy. This technique, which is based on the detection of two threshold electrons (kinetic energies $\leqslant 20 \mathrm{meV}$ ), has been named TPEsCO : threshold photoelectron(s) coincidence experiments. Finally in the third section the possibility of using high energy (hv,2e) experiments to extract direct informations on electron-electron correlations in the neutral initial state of the target will be addressed.

The photon energy range needed for these studies is from $\approx 30 \mathrm{eV}$ (where the $\mathrm{IP}^{2+}$ of polyatomic molecules are located) up to a few hundreds of eV to study the electronelectron correlation in the target. The only photon source tunable over such a broad range with reasonable flux to allow the collection of coincidence spectra within the experimentalist's lifetime is a synchrotron radiation source. Thus the TPEsCO studies were done at the Synchrotron Radiation Source (SRS)-Daresbury (UK), while the high energy (hv,2e) experiments are a challenge for third generation synchrotron radiation sources, like Elettra at Trieste or ALS at Berkeley.

\section{TPESCO INVESTIGATION OF DOUBLE IONIZATION IN RARE GASES}

The dynamics of two electrons simultaneously ejected by an atom due to absorption of a single photon is one of the fundamental processes of atomic physics. It requires a solution of the three-body Coulomb problem where the boundary conditions for the two electrons have to be included. The physical concepts governing this process were first discussed in a pioneering work by Wannier ${ }^{3}$ ). Since then considerable effort has been put, on the one hand, into extending this theory or developing quantal analogue and, on the other hand, experimentally verifying the predictions and determining their range of validity (an exhaustive review has been published by Read (4)).

Helium is the prototype system for the photo-double ionization process although its study is hampered by the particularly low cross-section. Nevertheless several observations have been made of the threshold behaviour a) of the photo-double ionization cross-section( ${ }^{5-8}$, b) of the energy distribution for the two outgoing electrons (6), and (c) of the angular distribution of these electrons (7.9). The heavier gases have received less attention because the study of the phenomena related to Wannier theory is hampered by the presence in the threshold region of indirect processes $\left({ }^{10}\right)$. 
Further theoretical analysis of the electrons in the Wannier geometry has shown that the symmetry of the electron pair wave functions can determine whether a final dicationic state is kinematically favoured or unfavoured. This "favouredness" depends whether the angular part of the electron pair wavefunction has a node or an antinode in the Wannier configuration(11-12). The rare gases are ideal for a study of this selectivity since they have several doubly charged states of different symmetry. For example, removal of two valence p electrons leads to ${ }^{3} \mathrm{P},{ }^{1} \mathrm{D}$ and ${ }^{1} \mathrm{~S}$ states of which the ${ }^{3} \mathrm{P}$ state would be favoured and the ${ }^{1} \mathrm{D}$ and $1 S$ unfavoured. PEPECO experiments are a suitable tool for investigating the final state selectivity because they allow a comparison of the relative cross-section for direct ionization to the different ionic states. To this purpose an existing apparatus (14) has been modified to enable the coincidence detection of energy selected electrons.

\subsection{Experimental method}

The main elements of the apparatus are a photon beam, an effusive gas beam and two electron energy analyzers, which are mounted on opposite sides of a turntable that can be rotated in a plane perpendicular to the photon beam, as shown in fig. 1 . The tunable photon beam, provided by the SRS via a toroidal grating monochromator, enters the apparatus via a $1 \mathrm{~mm}$ diameter glass capillary where it intersects the gas emanating from a hollow needle. The energy spread of the photon beam was typically $60-100 \mathrm{meV}$ and the photon flux was about $5 \times 10^{10}$ photons $/ \mathrm{sec}$.

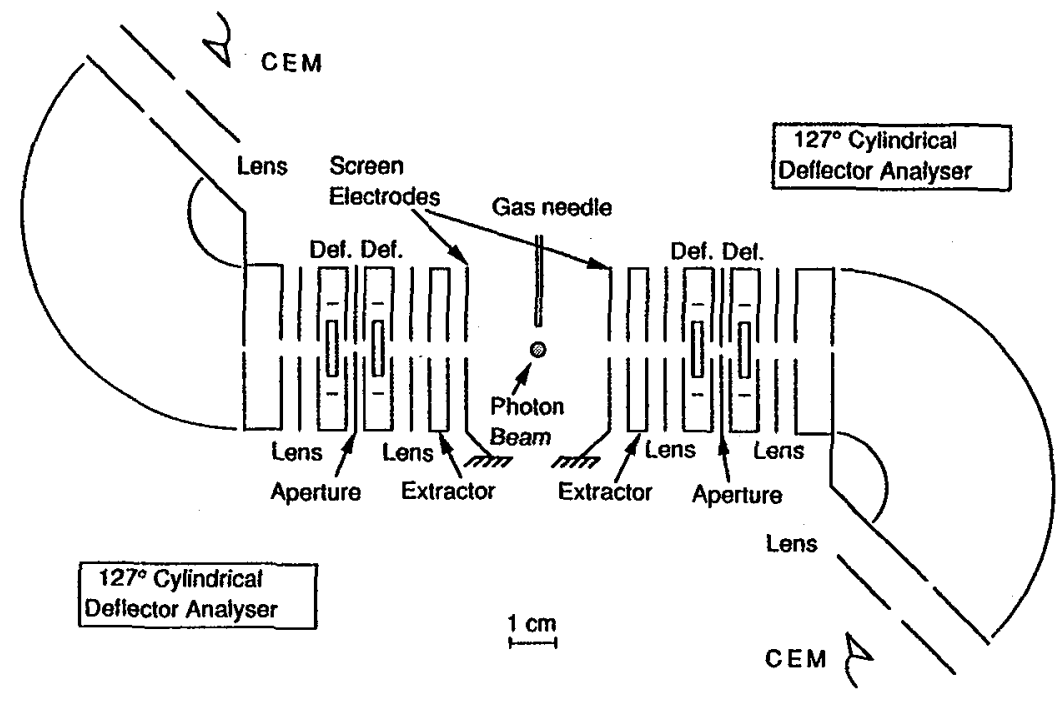

Figure 1 : Schematic diagram of the TPEsCO spectrometer

The coincidence spectrometer consists of a pair of $127^{\circ}$ cylindrical deflector analysers (CDA) developed from the photoelectron analyzer described by King et al. (15). Each of them is tuned to collect zero energy electrons using the penetrating field technique introduced by Cvejanovic and Read (16). Briefly a potential well is formed in the interaction region by field penetration of an extracting electrode, through a $5 \mathrm{~mm}$ aperture in a screening electrode. This provides a very large collection angle and furthermore forms a crossover point in the particle trajectories which ensures efficient trasmission of 
the electrons through the subsequent optics, which images the crossover onto the entrance plane of the CDA.

Standard NIM electronics is used to measure coincidences between electrons detected by the two analyzers. True and random coincidences and also the single counts, i.e. the photoelectron yield measured by each analyzer, are accumulated using a LSI11 minicomputer that also controlls the photon energy scan.

The collection modes of the analyzer were set up by balancing the extractor potentials (and the needle potential) in such a way that at the threshold of a photoionization process the electron yield was divided between the two analyzers in the ratio corresponding to their efficiencies. Those efficiencies were determined by maximizing, in turn, the threshold electron signal in one analyzer in the absence of the extractor potential of the other. Under these conditions the electrostatic potential between the analyzers has the form of a symmetric saddle. In close vicinity of the threshold photo-double ionization yields two near zero energy electrons, which when emitted in approximately opposite directions, can drift to different sides of the potential ridge and be detected in different analyzers. A true coincidence signal is then the signature of a double ionization event and will be recorded each time the scanned photon energy goes through the threshold of a double ionization state. A spectrum obtained in this way is then a TPESCO spectrum and is the double ionization analogue of a threshold electron spectrum used in the study of single ionization processes. The threshold energy resolution of the two analyzers is less than 20 $\mathrm{meV}$ so that the heights of the observed peaks correspond to the relative cross-sections for excitation of the doubly charged states within $20 \mathrm{meV}$ of their respective thresholds. The two analyzers collect threshold photoelectrons over a solid angle of approximately $2 \pi$ steradians and so the spectra presented do not provide any angular information on the cross sections.

\subsection{Results}

TPEsCO spectra have been measured for all the rare gases from neon to xenon in an energy region which includes the doubly charged states $n p^{4}\left({ }^{3} P,{ }^{1} \mathrm{D}\right.$ and $\left.{ }^{1} \mathrm{~S}\right)$ and, with the exception of xenon, the $n s n p^{5}$ ( $3 \mathrm{P}$ and $1 \mathrm{P}$ ) states, as well. As an example in fig. $2 \mathrm{a}$ and $2 \mathrm{~b}$ the TPEsCO spectra of $\mathrm{Ne}$ and $\mathrm{Ar}$ are shown. The observed energies of these states are in agreement with the spectroscopic values (17-21). Counting rates were typically a few counts per second and the true to random coincidence ratios were typically 20:1. The energy resolution of $\approx 75 \mathrm{meV}$, mainly due to the energy spread of the photon beam, allows the separation of the double ion states and of the spin orbit components of some ${ }^{3} \mathrm{P}$ states.

The first important point of note in the spectra of fig. 2 is that all the possible ionic states are observed. This finding is in good agreement with the studies of Price and Eland(22-23) in argon and xenon, but in disagreement with the results of Lablanquie (24) who observed exclusively the formation of the ${ }^{3} \mathrm{P}$ ground state of argon. The disagreement with the latter work may be due to the limited statistical accuracy of their data and also to the fact that the observations were made more than $10 \mathrm{eV}$ above threshold.

As far as the relative intensity is concerned, it appears that the unfavoured states are excited with intensities that are not very different from the favoured ones. Greene and Rau $(11,25)$ have pointed out that two electron excitations are most probable when the LSח quantum numbers of the electron pair are either all even or else all odd. For example photoionization from the $1 \mathrm{Se}$ ground state to the $3 \mathrm{Pe}$ doubly charged state is favoured because the electron pair in the ${ }^{3} \mathrm{po}^{\circ}$ state has all odd values. On the other hand the ${ }^{1} \mathrm{D}^{\mathrm{e}}$ and $1 \mathrm{Se}$ doubly charged states have states of the electron pair ( $\left.{ }^{1} \mathrm{Po},{ }_{1}^{1} \mathrm{Do},{ }_{1 \mathrm{FO}} . ..\right)$ none of which are either all odd or all even and therefore are unfavoured. Huetz et al. (13) in their 


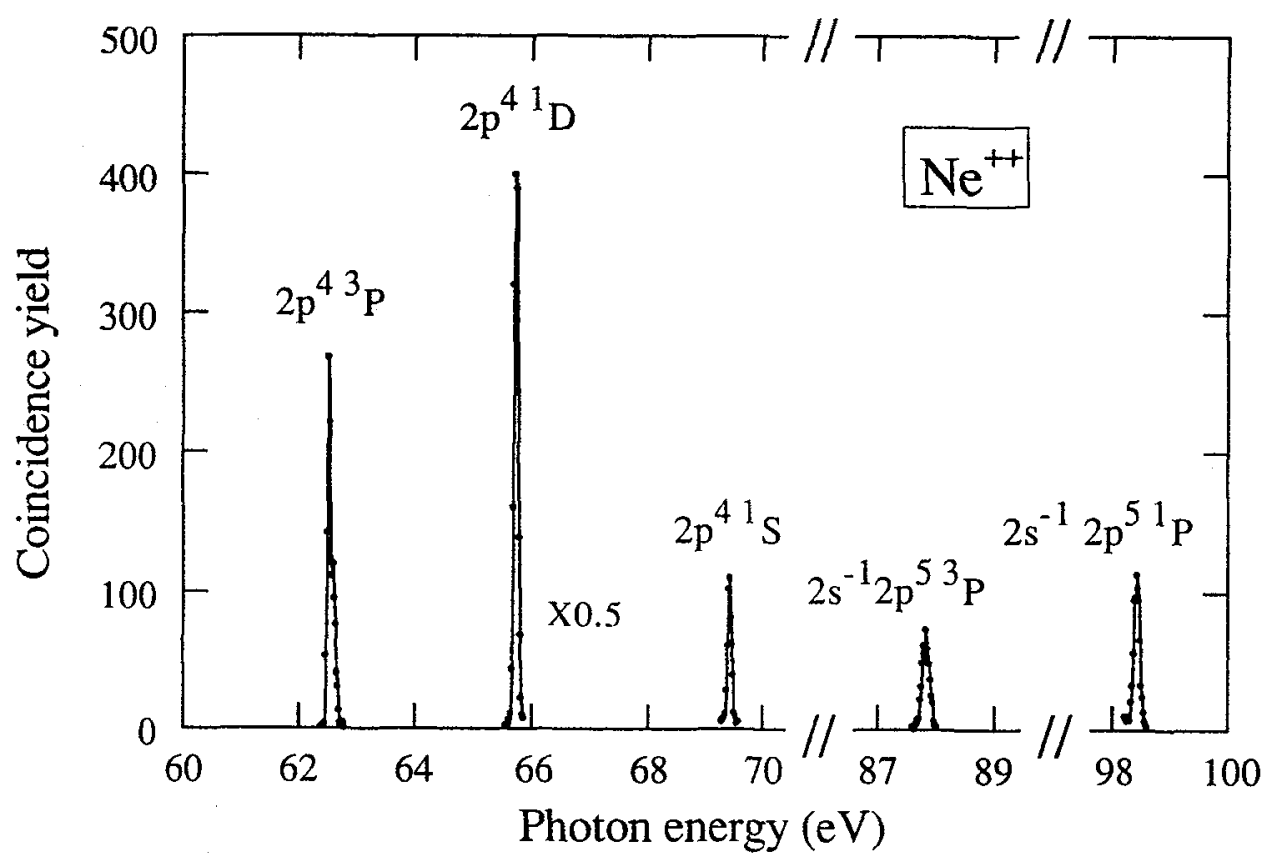

Figure 2a: TPEsCO spectrum of Ne over the photon energy range 62 to $100 \mathrm{eV}$

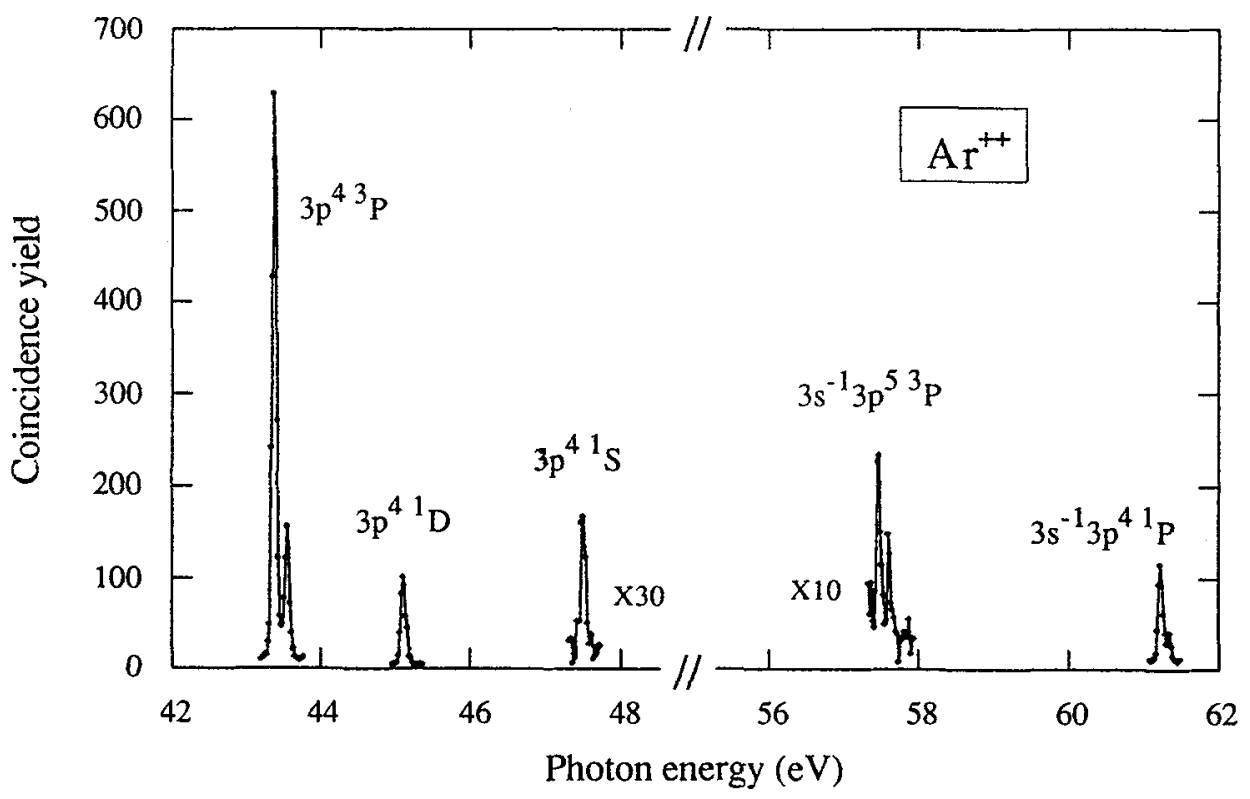

Figure $2 \mathrm{~b}$ TPEsCO spectrum of Ar over the photon energy range 42 to $62 \mathrm{eV}$ 
extension of the Wannier theory deduced an angular form factor for the cross-sections of doubly charged states which is smaller, but none vanishing, for the unfavoured final states, compared to the favoured cases. According to their model the hierarchy of "favouredness" leads to a decreasing intensity from $3 \mathrm{P}$ to ${ }^{1 D}$ to $1 \mathrm{~S}$ states. Our observations are in general accord with this propensity rule. Indeed all the states are observed, and in $\mathrm{Ar}$ (fig. 2b) , $\mathrm{Kr}$ and $\mathrm{Xe} \mathrm{(26)} \mathrm{the}{ }^{3} \mathrm{P}$ ion peaks do have the largest intensity. The exception to this is $\mathrm{Ne}$ (fig. 2a), where the ${ }^{1} \mathrm{D}$ peak has the largest intensity.

Indirect excitation of doubly charged states via the decay of satellite states could influence the intensities observed in PEPECO spectra. The TPEPICO study of Hall et al. (10) showed that a characteristic of the satellite states in rare gases is that they decay solely to the double ion continuum once this channel is energetically open. However in the present work only satellite states within $20 \mathrm{meV}$ of the ${ }^{3 \mathrm{P}},{ }^{1} \mathrm{D},{ }^{1} \mathrm{~S}$ thresholds can contribute to the observed intensity. Hall et al. (27) in their high resolution investigation of the $\mathrm{Ne}$ satellites have shown that all three dication states of $\mathrm{Ne}$ appear to be free from any intense satellite structure. Therefore the cross-sections measured in the TPEsCO spectrum should correspond to those for the direct process. Thus to date the anomalous behaviour of $\mathrm{Ne}$ is an open question.

\section{SPECTROSCOPY OF MOLECULAR DICATIONS USING THE TPESCO TECHNIQUE}

Doubly charged ions of diatomic molecules have been known to exist for several decades (28). The ground states of these molecules are characterized by potential curves (fig. 3) with a local minimum, produced by the binding of the valence electrons. At larger internuclear separations the Coulomb repulsion between the two positively charged ions dominates and the system dissociates to a limit which is always lower in energy than the local minimum. The height of the Coulomb barrier between these regions determines the lifetimes of the species that sometimes are so long that the ion can be considered stable.

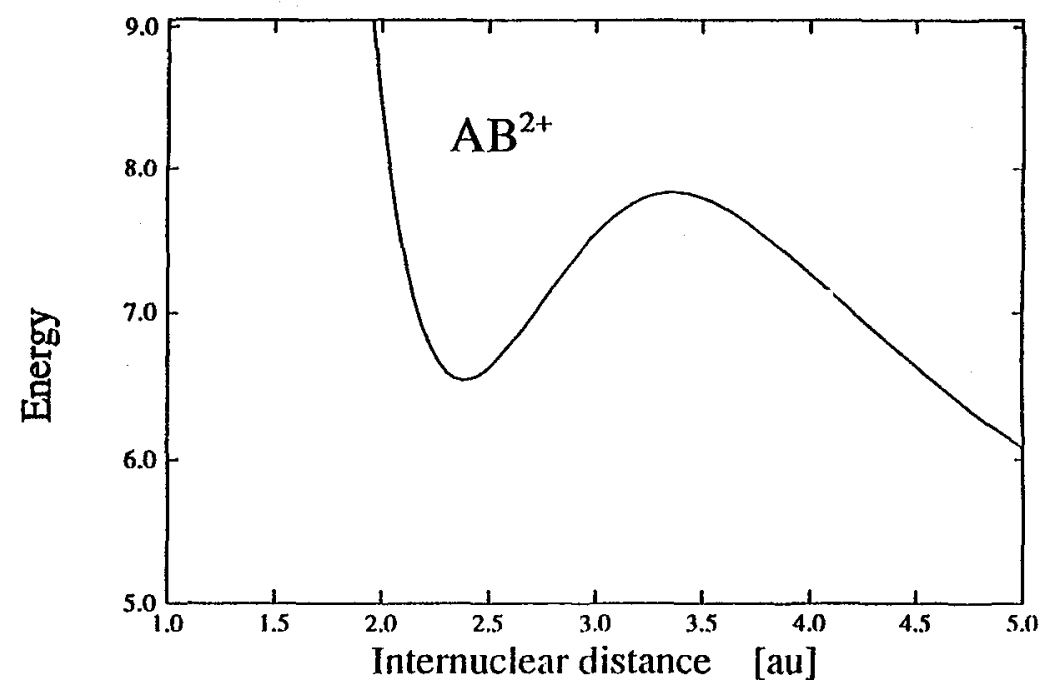

Figure 3 : Potential energy curve for a diatomic dication $\mathrm{AB}^{2+}$. The energy scale in $\mathrm{eV}$ refers to the dissociation limit $\mathrm{A}^{+}+\mathrm{B}^{+}$. 
Since their discovery, very little precise experimental information has been obtained on the electronic states of molecular dications. A whole variety of techniques ( see for istance (29) and reference therein) have been applied to their study. The high resolution techniques, such as ion beam laser spectroscopy are applicable only over a limited energy range, while those that cover a broad energy range, like for example energy gain spectroscopy or photofragmentation spectroscopy, lack energy resolution. In several molecules a fundamental quantity like the double ionization threshold is either unknown or known with a large uncertainty.

The TPEsCO technique can tackle the investigation of doubly charged ions directly, as shown by the first ever measurement by Hall et al. $\left({ }^{30}\right)$ with a preliminary apparatus on $\mathrm{N}_{2}$ and $\mathrm{CO}$. The main advantage of a PEPECO experiment consists in the fact that the observations are independent of the subsequent "fate" of the dication.

In fig. $4 \mathrm{a}$ and $4 \mathrm{~b}$ the TPEsCO spectrum of nitrous oxide over the energy range $38-45 \mathrm{eV}$ is shown. The total accumulation time for this spectrum was 32 hours so that each data point taken at $20 \mathrm{meV}$ intervals, had a total dwell time of 5.5 minutes. The coincidence count rate on the first peak of fig.4a was about 1.3 counts $/ \mathrm{sec}$ and the true to random ratio was approximately 9:1. The energy scale of the spectra have been obtained using a mixture of $\mathrm{Ar}$ and NO and the features in the NO TPESCO spectrum are calibrated against the known $\mathrm{Ar}^{2+} 3 \mathrm{p}^{4}\left({ }^{3} \mathrm{P}_{2}\right)$ threshold. The peak widths in these spectra represent an overall instrumental resolution of $80 \mathrm{meV}$, which corresponds to the energy spread of the photon beam.

Table 1 : Appearance energy for the $\mathrm{X}^{2} \Sigma^{+}$

$\begin{array}{lll}\text { Method } & \text { (Year) Ref. } & \text { A.P. }(\mathrm{eV}) \\ \text { double charge transfer } & (1973) 50 & 39.3 \pm 0.5 \\ & (1987) 56 & 38.6 \pm 0.3 \\ \text { Auger spectroscopy } & (1971) 51 & 35.1-37.0 \\ & (1992) 32 & 38.55 \pm 0.1 \\ \text { mass spectrometry } & (1981) 52 & 39.3 \pm 0.3 \\ & (1961) 53 & 39.8 \pm 0.3 \\ & (1966) 54 & 38.3 \pm 0.5 \\ & (1986) 55 & 38.6 \pm 0.1 \\ \text { ion photofragment spectr. } & (1992) 32 & 38.6 \pm 0.3 \\ \text { present work } & & 38.47 \pm 0.02\end{array}$

Fig.s 4 clearly show that both the onset for double ionization and the vibrational structure of the dication electronic states can be obtained directly from the TPEsCO spectra. This technique along with the recently performed Double ZEKE experiments ( ${ }^{31}$ ), provides the only direct methods to date of making such observations. The first feature in the spectrum was located at $38.47 \pm 0.02 \mathrm{eV}$. This value is in agreement with, but definitely more accurate than, the more recent determinations of the appearance potentials of $\mathrm{NO}^{2+}$ as shown in Tab. 1 .

NO has a ground configuration $1 \sigma^{2} 2 \sigma^{2} 3 \sigma^{2} 4 \sigma^{2} 1 \pi^{4} 5 \sigma^{2} 2 \pi^{12} \Pi$, therefore the first $\mathrm{NO}^{2+}$ states are obtained by removing two electrons from the $2 \pi$ and $5 \sigma$ orbitals ( $\mathrm{NO}^{2+}$ ground state $X^{2} \Sigma$ ), from the $2 \pi$ and $1 \pi$ orbitals (first excited state $A^{2} \Pi$ ) and from the $2 \pi$ and $4 \sigma$ orbitals $\left(B^{2} \Sigma\right.$ state). These are the states that are expected to be excited within the range covered by the present experiment. Thus the observed features have been assigned as vibrational progressions of these three electronic states $\mathrm{NO}^{2+}$.

The double ionization energy for the $A$ and $B$ states have been located at $39.94 \pm 0.02$ and $43.29 \pm 0.02 \mathrm{eV}$; both the values are in good agreement with the ones $(40.1 \pm 0.1$ and $43.3 \pm 0.1$ 


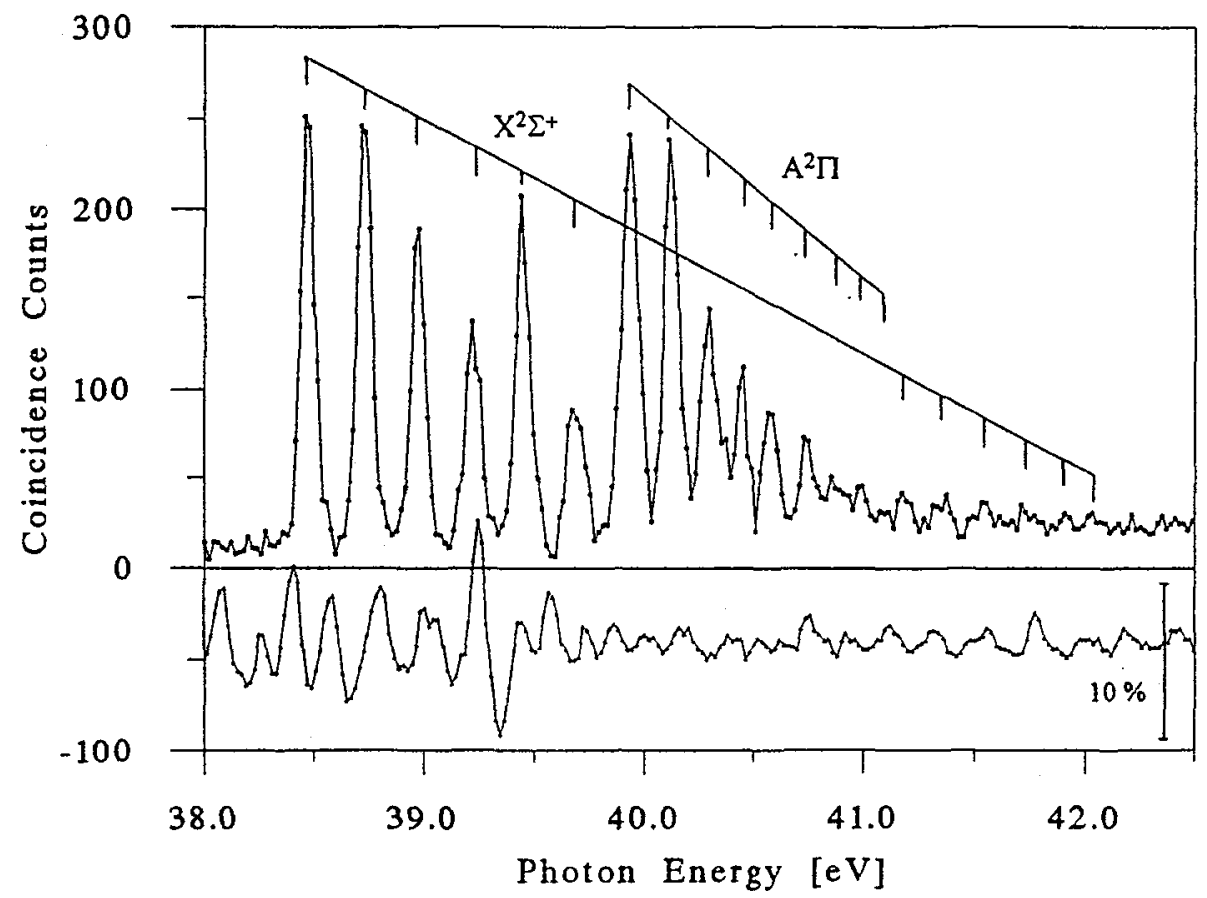

Figure 4a: TPEsCO spectrum of $\mathrm{NO}^{2+}$ over the photon energy range 38 to 42.5 $\mathrm{eV}$. The lower spectrum in the figure is the sum of the threshold spectra collected in both analyzers.

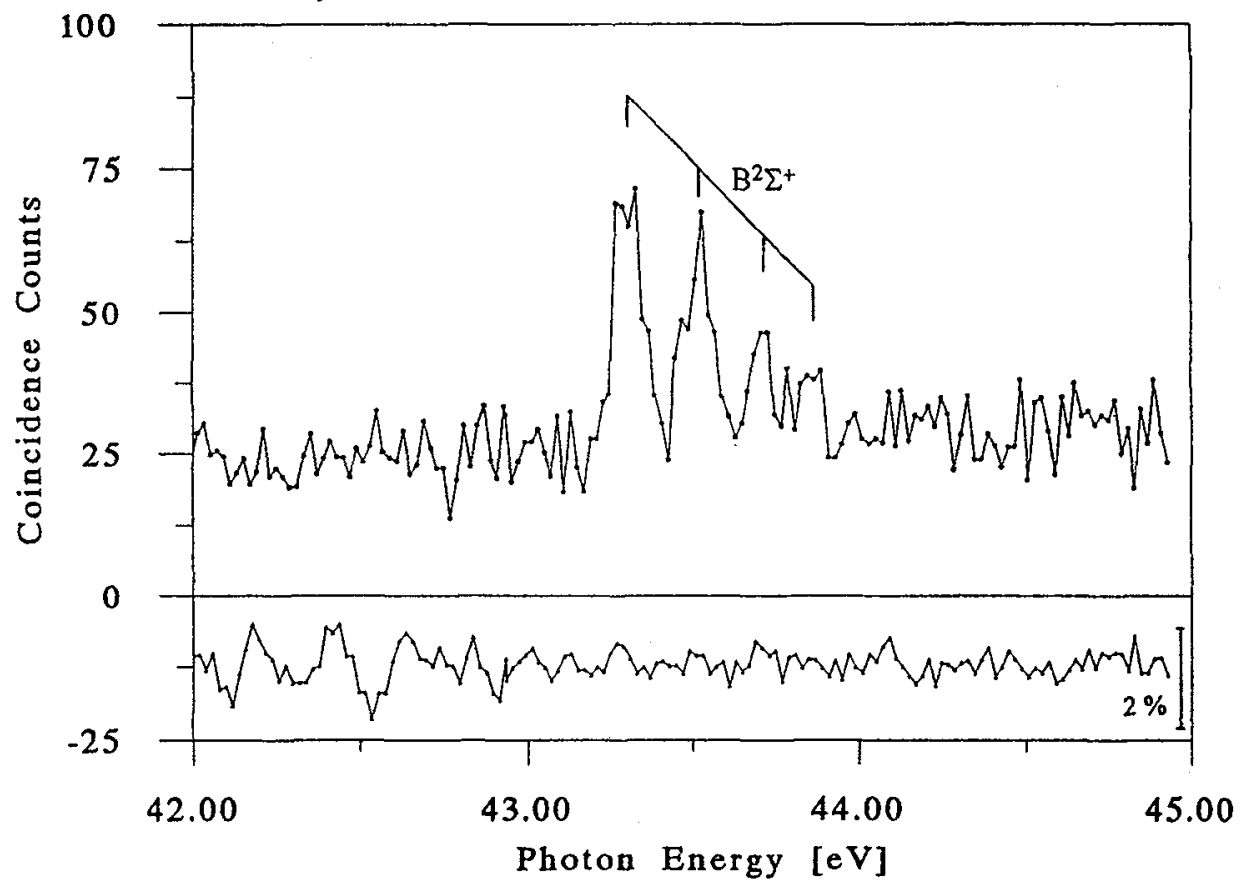

Figure 4b: TPEsCO spectrum of $\mathrm{NO}^{2+}$ over the photon energy range 42 to $45 \mathrm{eV}$. 
eV) obtained from an Auger experiment by Petterson et al. (32). The vibrational motion of the lowest levels of the dications of diatomic molecules is well represented by that of an anharmonic oscillator, thus the expression

$$
E(v)=T_{0}+\omega_{e}(v+1 / 2)-\omega_{e} x_{e}(v+1 / 2)^{2}
$$

where $T_{0}$ is the value of the potential minimum referred to the $v=0$ level of the NO ground state and $\omega_{e}$ and $\omega_{e} x_{e}$ are the vibrational constants, has been fitted to the observed energies of the vibrational levels of the different electronic states. The sets of molecular constants for the three states are reported in Tab. 2 together with the ones predicted by the theoretical calculations of Wetmore and Boyd (33) and Petterson et al. (34). The more recent values of Petterson et al. (34) compare quite favourably with those observed in the present work.

Table 2: Molecular constants

\begin{tabular}{|lllll|}
\hline \multirow{3}{*}{$\mathrm{X}^{2} \Sigma^{+}$} & $\mathrm{T}_{0}(\mathrm{eV})$ & $38.39 \pm 0.05$ & 39.42 & 38.56 \\
& $\omega_{\mathrm{e}}(\mathrm{meV})$ & $255 \pm 19$ & 277 & 261 \\
& $\omega_{\mathrm{e}} \mathrm{x}_{\mathrm{e}}(\mathrm{meV})$ & $3 \pm 1$ & 4 & 3 \\
$\mathrm{~A}^{2} \Pi$ & $\mathrm{T}_{0}(\mathrm{eV})$ & $39.85 \pm 0.007$ & 39.96 & 40.02 \\
& $\omega_{\mathrm{e}}(\mathrm{meV})$ & $186 \pm 4$ & 170 & 184. \\
& $\omega_{\mathrm{e}} \mathrm{x}_{\mathrm{e}}(\mathrm{meV})$ & $4.5 \pm 0.4$ & 3 & 4.6 \\
$\mathrm{~B}^{2} \Sigma^{+}$ & $\mathrm{T}_{0}(\mathrm{eV})$ & $43.16 \pm 0.015$ & & \\
& $\omega_{\mathrm{e}}(\mathrm{meV})$ & $272 \pm 18$ & & \\
& $\omega_{\mathrm{e}} \mathrm{x}_{\mathrm{e}}(\mathrm{meV})$ & $20 \pm 4$ & & \\
& & & & \\
\end{tabular}

Further information which can be extracted from the TPESCO spectrum is related to the mechanism of formation of doubly charged ion. The absorption of an energetic photon can result in direct emission of two electrons, or in ionization to an excited state of the singly charged ion, that may autoionize to a lower lying dication state. This latter two step process has been observed to play a major role in double ionization of rare gases near threshold in the TPEPICO experiments $(10)$. In the TPEsCO spectrum the indirect mechanism will only contribute if the single ion state is excited within $20 \mathrm{meV}$ of its threshold and then if it lies within $20 \mathrm{meV}$ of the double ion state. The intensities of the experimental peaks give indications of the relative importance of the two mechanisms. If only the direct mechanism were to occur, the peaks in the various vibrational series would have intensities governed by the Franck-Condon principle. The indirect mechanism, on the other hand, results in erratic peak envelopes with intensities governed by a combination of the Franck-Condon principle for the excitation of the single ion state and the cross-section for the autoionization process. In order to ascertain the contribution of the indirect mechanism the sum of the threshold spectra taken by both the analyzers is shown in the bottom part of fig. 4 . In this way a direct comparison is then possible in order to establish whether any energy degeneracy exists and thus indicates the possible role of the indirect mechanism. In the NO case the direct mechanism appears to be the dominant mechanism, except for the region $v=0-4$ of the $X$ state, where some structures are present in the threshold spectrum of the singly charged ion which enhance the $v=4$ level and produce a shoulder in the $v=3$ peak. This case is quite different from the $\mathrm{O}^{2+}$ one (35) where the two step process has been shown to result in the excitation of high vibrational levels outside the Franck-Condon region. 
The results for NO, as well as those for several other diatomic (36-37) and polyatomic (38) molecules, show that TPEsCO has unique performances in providing accurate double ionization energies, vibrational constants for the different electronic states of the dications and useful informations on the possible formation mechanisms. A further step in the investigation of dications will consist in experiments which follow the fragmentation pattern of the doubly charged ions after a detailed selection of the parent doubly charged ion via TPESCO.

\section{BACK TO THE FUTURE : DOUBLE PHOTOIONIZATION AT HIGH ENERGY}

Since the beginning of their development electron-electron coincidence experiments in electron impact ionization, i.e. $(e, 2 e)$ experiments, have attracted a lot of interest because they were expected to be a useful tool to investigating the electronic structure of the target particle. Indeed whenever the ionizing reaction can be described within the impulse approximation, i.e. one can assume that the energy and momentum transfer in the reaction are adsorbed by a single target electron, then the triple differential cross-section factorizes in two terms

$$
\frac{d^{3} \sigma}{d E d \Omega_{1} d \Omega_{2}}=4 \frac{k_{s} k_{e}}{k_{0}} f_{e e}^{t} \rho(q)
$$

where $k_{0}, k_{s}$, and $k_{e}$ are the momenta of the incident, scattered and ejected elctrons, respectively. The half off-shell Mott scattering term $\mathrm{ft}_{\mathrm{t}}$ ee describes the electron interaction

$$
\begin{aligned}
& \mathrm{f}_{\mathrm{ee}}^{\mathrm{t}}=\mathrm{C}_{0}^{2}(\eta)\left\{\frac{1}{\mathrm{~K}^{4}}+\frac{1}{\mathrm{Q}^{4}}-\cos \left[\eta \ln \left(\frac{\mathrm{Q}^{2}}{\mathrm{~K}^{2}}\right)\right] \frac{1}{\mathrm{Q}^{2} \mathrm{~K}^{2}}\right\} \\
& \mathrm{C}_{0}^{2}(\eta)=2 \pi \eta /[\exp (2 \pi \eta)-1], \eta=1 / \mathrm{K}^{\prime}
\end{aligned}
$$

where $K=\mathbf{k}_{\mathbf{0}}-\mathbf{k}_{\mathbf{s}}, \mathbf{Q}=\mathbf{k}_{0}-\mathbf{k}_{\mathbf{e}}$, and $K^{\prime}=1 / 2\left(\mathbf{k}_{\mathbf{e}}-\mathbf{k}_{\mathbf{s}}\right)$. The form factor $\rho(q)$, where $q$ is the momentum of the bound electron determined by the momentum conservation $\mathbf{k}_{\mathbf{0}}+\mathbf{q}=\mathbf{k}_{\mathbf{s}}$ $+k_{e}$ is the squared transform of the overlap integral between the initial state of the target and the final state of the ion.

$$
\begin{aligned}
& \rho(\mathrm{q})=\left|\left\langle q \psi_{\mathrm{f}} \mid \psi_{\mathrm{i}}\right\rangle\right|^{2}= \\
& \left|\int \exp \left(-\mathrm{iq} \cdot \mathrm{r}_{\mathrm{i}}\right) \mathrm{d} \mathrm{r}_{\mathrm{i}} \int \psi_{\mathrm{f}}^{*}\left(\mathrm{r}_{1} \cdot \mathrm{r}_{\mathrm{i}-1} \mathrm{r}_{\mathrm{i}+1} \cdot \mathrm{r}_{\mathrm{N}}\right) \psi_{\mathrm{i}}\left(\mathrm{r}_{1} \cdot \mathrm{r}_{\mathrm{i}} \cdot \mathrm{r}_{\mathrm{N}}\right) \mathrm{dr}_{1} . . \mathrm{dr} \mathrm{r}_{\mathrm{i}-1} \mathrm{~d} \mathrm{r}_{\mathrm{i}+1} \cdot \mathrm{d} \mathrm{r}_{\mathrm{N}}\right|^{2}
\end{aligned}
$$

In the independent-particle frozen-orbital approximation $\rho(q)$ reduces to the single electron momentum density of the ionized orbital. The validity of the impulse approximation has been established in several different kinematics $(39,40)$ and a large number of atomic and molecular orbitals have been studied via $(e, 2 e)$ reactions $(41)$.

In 1977 Neudatchin et al.(42) proposed the high energy $(\mathrm{hv}, 2 \mathrm{e})$ coincidence experiments to investigate the double ionization of atoms and molecules. These experiments should represent the natural generalization of impulsive $(e, 2 \mathrm{e})$ experiments. The goal of the proposal (42-44) was to develop a spectroscopy of doubly charged ions that would enable the direct determination of the partial two-electron Fourier amplitude 


$$
\psi_{\text {if }}\left(\mathrm{k}_{1}, \mathrm{k}_{2}\right)=\frac{1}{2 \pi} \int \mathrm{dr}_{1} \mathrm{dr}_{2} \psi_{\text {if }}\left(\mathrm{r}_{1}, \mathrm{r}_{2}\right) \mathrm{e}^{\mathrm{i}\left(\mathrm{k}_{1} \cdot \mathrm{r}_{1}+\mathrm{k}_{2} \cdot \mathrm{r}_{2}\right)}
$$

of the many-electron wavefunction $\psi_{i}\left(\mathbf{r}_{1}, \mathbf{r}_{2}, \ldots \ldots . . \mathbf{r}_{N}\right)$ projected on an arbitrarily selected state $\psi_{\mathrm{f}}\left(\mathbf{r}_{3}, \mathbf{r}_{4}, \ldots \ldots . \mathbf{r}_{\mathrm{N}}\right)$ of the doubly charged ion

$$
\psi_{\text {if }}\left(r_{1}, r_{2}\right)=\int d r_{3} . . d r_{N} \psi_{f}^{*}\left(r_{3}, \ldots r_{N}\right) \psi_{i}\left(r_{1}, \ldots r_{N}\right)
$$

The importance of studying these quantities becomes clear when a two electron system is considered. In this case $\psi_{\mathrm{if}}\left(\mathbf{k}_{1}, \mathbf{k}_{2}\right)$ is merely the wave function of the two electron system in the momentum space representation. It contains all the details of the structure of the system and in particular the momentum distribution of the relative motion of the electrons in the degree of freedom $\mathbf{r}_{12}=\mathbf{r}_{1}-\mathbf{r}_{2}$. This motion is dominated by electronelectron correlations. In many-electron systems if the transition $\mathrm{i} \rightarrow \mathrm{f}$ involves the knockout of two electrons from the same orbital then $\psi_{i f}\left(\mathbf{k}_{1}, \mathbf{k}_{2}\right)$ characterizes the electronelectron correlations in that orbital, viceversa if the transition involves electrons from different orbitals then $\psi_{\mathbf{i f}}\left(\mathbf{k}_{1}, \mathbf{k}_{2}\right)$ enables the study of electron-electron correlations in different orbitals. The sensitivity of the technique can be inferred from a theoretical study of the double photoionization of the $\mathrm{H}_{2}$ molecule (44). Under the assumption that the energies of both the final electrons are large compared with their binding energies in the target, these authors calculated the triple differential cross-section by using different wave functions for the $\mathrm{H}_{2}$ ground state. Depending on the choice of the wavefunctions the cross-section showed completely different shapes. Thus such a high energy experiment is expected to provide an "ideal" manner to reconstruct the electron-electron correlator in the wavefunction from the experimental data.

\subsection{Present feasibility and future of high energy (hv,2e)}

Let's now investigate the feasibility of such an experiment on a target with known double ionization cross-section $\sigma^{2+}$, like $\mathrm{He}$ or another rare gas. Holland et al. (45) have measured $\sigma^{2+}$ for the rare gases up to $280 \mathrm{eV}$ photon energy. From their data $\sigma^{2+}$ seems to peak in the region $100-150 \mathrm{eV}$ and the absolute value varies from $0.2 \mathrm{Mb}(\mathrm{He})$ to $7 \mathrm{Mb}(\mathrm{Xe})$. In order to estimate the expected coincidence rate in the PEPECO experiment we have assumed an uniform energy distribution of the emitted electrons and an isotropic angular distribution. With these assumptions the $\mathrm{d}^{3} \sigma / \mathrm{dE} \mathrm{E}_{1} \mathrm{~d} \Omega_{1} \mathrm{~d} \Omega_{2}$ at $\mathrm{hv}=150 \mathrm{eV}$ has the values of about $2 \times 10^{-23}$ and $4 \times 10^{-22} \mathrm{~cm}^{2} / \mathrm{eV}$ ster in $\mathrm{He}$ and $\mathrm{Xe}$, respectively. The coincidence count rate can be estimated from the relation

$$
\mathrm{N}_{\mathrm{c}}=\Phi \cdot(\mathrm{nl}) \cdot \frac{\mathrm{d}^{3} \sigma}{\mathrm{dEd} \Omega_{1} \mathrm{~d} \Omega_{2}} \cdot \Delta \mathrm{E} \Delta \Omega_{1} \Delta \Omega_{2} \varepsilon_{1} \varepsilon_{2}
$$

where $\Phi$ is the photon flux, $\mathrm{n}$ and $\mathrm{l}$ the target density and length respectively, $\Delta \mathrm{E}$ the coincidence energy resolution, $\Delta \Omega_{i}$ and $\varepsilon_{i}$ the accepted solid angle and efficiency of each spectrometer. In a typical gas-phase experiment at the Daresbury-SRS the target density is about $10^{13}$ atoms $/ \mathrm{cm}^{3}$ with a target length of about $1 \mathrm{~mm}$ and the flux on the target is in the order of a few $10^{10}$ photons/sec. By using these values and assuming $\Delta \mathrm{E}=1 \mathrm{eV}$, $\Delta \Omega_{\mathrm{i}}=0.1$ ster and $\varepsilon_{\mathrm{i}}=1$ one obtains $4 \times 10^{-2}$ counts $/$ sec in the more favourable case. Despite 
the fact that an analogous estimation of the random coincidence rate $N_{f}$, results in values about $5 \times 10^{-4}$ counts/ sec and therefore $N_{c} / N_{f} \gg 1$, the time needed to obtain a single experimental point with statistics of $10 \%$ is larger than 2 hours. This makes such an experiment unrealistic for actual synchrotron radiation sources and "conventional" electron-electron coincidence spectrometers.

On the other hand, the advent of third generation synchrotron radiation sources together with the development of multidetection and multicoincidence techniques are expected to make possible the investigation of high energy (hv,2e) (46).

The main characteristic of the third generation synchrotron radiation sources consists in the fact that the synchrotron radiation will be mainly produced by insertion devices, devoted to enhance the brilliance and the photon flux in a certain energy region. In the case of Elettra, for example, the insertion device designed for the gas phase beam-line is a $11.5 \mathrm{~cm}$ period pure permanent magnet undulator, which covers the energy region 20-800 $\mathrm{eV}\left({ }^{47}\right)$. Similar devices are planned also for ALS at Berkeley (48). The flux on the sample from such devices is expected to be about $10^{12}$ photons/sec at $0.1 \%$ bandwith. This flux, which is from 50 to 100 times higher than the one from a bending magnet of a second generation source, will already constitute a considerable improvement. However in order to tackle the high energy $(\mathrm{h} v, 2 \mathrm{e})$ experiments in a more effective way, the construction of spectrometers that accept the largest possible solid angle without loosing in energy and angular selectivity becomes a must. These new apparatuses must allow the simultaneous detection of the electron pairs whichever is the relative emission angle in or out the polarization plane of the incident light. Only in this way it is possible to map completely the triple differential cross-section and to extract the information on the electron-electron correlations in the initial state of the target.

At present several group in Australia (49), USA (57) and Europe (58) are pursuing this goal. This new generation of spectrometers will found several other applications that extend from multi-parameter $(e, 2 e)$ and $(e, 3 e)$ experiments to Auger-photoelectron coincidence experiments on both gas phase and solid targets.

\section{Acknowledgments}

Work partially supported by a CNR(Italy)-ROYAL SOCIETY (UK) exchange programme and by the NATO grant C.R.G.920101

\section{References :}

[1] Schmidt V., "Double Ionization Coincidence Spectroscopy ", in X-ray and Inner Shell Processes, T.A. Carlson, M. O. Krause, and S.T. Manson, AIP Conference Proceedings 215 (1990),pp. 559-581

[2] Eland J. H. D., "Coincidence Studies of Multiionized Molecules " in Vacuum Ultraviolet Photoionization and Photodissociation of Molecules and Clusters ", Ed. C.Y. Ng (World Scientific, London 1991),pp. 297-343

[3] Wannier G. H., Phys. Rev. 90 (1953), 817

[4] Read F. H., "Threshold behaviour of Ionization Cross-Section", in Electron Impact Ionization ,Ed.s T.D. Mark and G.H. Dunn ( Springer-Verlag Wien 1992),pp. 42-89

[5] Kossman H., Schmidt V, Andersen T., Phys. Rev. Lett. 60 (1988), 1266

[6] Lablanquie P., Ito K., Morin P., Nenner I., Eland J.H.D., Z. Phys. D16 (1990), 77

[7] Hall R.I. , McConkey A.G., Avaldi L., Ellis K., MacDonald M.A., Dawber G. and King G.C., J. Phys. B25 (1992), 1195

[8] Hall R.I. , Avaldi L., Dawber G., Zubek M., Ellis K., and King G.C., J. Phys. B24 (1991), 1917

[9] Schwarzkorpf O., Krassig B., Elminger J. and Schmidt V. , Phys. Rev. Lett. 70 (1993) , 3008 
[10] Hall R.I. , Ellis K.,McConkey A.G., Dawber G.,Avaldi L., MacDonald M.A., and King G.C., J. Phys. B25 (1992), 377

[11] Greene C. H. and Rau A. R. P. , Phys. Rev. Lett. 48 (1982), 533

[12] Stauffer A. D., Phys. Lett. 91A (1982), 114

[13] Huetz A., Selles P., Waymel D., Mazeau J., J. Phys. B24 (1991), 1917

[14] Hall R.I. , McConkey A.G., Ellis K., Dawber G., Avaldi L., MacDonald M.A., and King G.C., Meas. Sci. Tech. 3 (1992), 316

[15] King G. C. , Zubek M., Rutter P. M. and Read F.H., J. Phys. E20 (1987),440

[16] Cvejanovic S. and Read F. H., J.. Phys. B7 (1974), 1180

[17] Moore C. E. , Atomic Energy Level, vol 1 (NBS Ref. Data Series 35) Washington DC(1971)

[18] Persson W., Phys. Scr. 3 (1971), 133

[19] Minnhagen L., Strihed H. and Petterson B., Ark. Fys. 34 (1969), 471

[20] Hansen J. E. , and Persson W., Phys. Scr. 25 (1981), 487

[21] Hansen J. E. and Persson W., Phys. Rev. A18 ( 1978), 1459

[22] Price S. D. and Eland J.H.D., J. Phys. B22 (1989), L153

[23] Price S. D. and Eland J.H.D., J. Elect, Spec. Rel. Phenom. 52 (1990), 649

[25] Greene C. H. and Rau A. R. P. , J. Phys. B16 (1983) , 99

[26] Hall R.I., Dawber G., McConkey A.G., MacDonald M.A. and King G.C., Z. Phys. D23 (1992),377

[27] Hall R.I. , Dawber G., Ellis K., Zubek M., Avaldi L. and King G.C., J. Phys. B24 (1991), 4133

[28] Vaugham A. L., Phys. Rev. 38 (1931),1687

[29] Larsson M, Comm. At. Mol. Phys. (1993) to be published

[30] Hall R. I., McConkey A. , Avaldi L., MacDonald M. A. and King G. C. ,J. Phys. B 25 (1992), 411

[31] Krassig B. and Schimdt V., J. Phys. B25 (1992), L327

[32] Petterson L.G.M., Karlsson L., Keane M. P. , Naves de Brito A., Correia N., Larsson M., Brostrom L., Mannervik S. and Svensson S., J. Chem. Phys. 96 (1992), 4884

[33] Wetmore R. W. and Boyd R.K., J. Phys. Chem.90 (1986), 6091

[34] Petterson L. G. M., Siegbahn P.E.M. , Brostrom L., Mannervik S. and Larsson M., Chem. Phys. Lett. 191 (1992), 279

[35] Hall R. I., Dawber G., McConkey A., MacDonald M. A. and King G.C., Phys. Rev. Lett. 68 (1992) 2751

[36] Hall R. I., Dawber G., McConkey A., Avaldi L., MacDonald M. A. and King G.C., submitted to J. Phys. B (1993)

[37] Hall R. I., Dawber G., McConkey A., Avaldi L., MacDonald M. A. and King G.C., submitted to J. Phys. B (1993)

[38] Hall R. I., Dawber G., McConkey A., Avaldi L., MacDonald M. A. and King G.C., to be submitted

[39] McCarthy I.E. and Weigold E., Rep. Prog. Phys. 54 (1991), 789

[40] Avaldi L., Camilloni R., Fainelli E. and Stefani G. , J. Phys. B20 (1987),4163

[41] Brion C., "Chemical application of $(\mathrm{e}, 2 \mathrm{e})$ reaction in electron momentum spectroscopy" in Correlation and Polarization in Electronic and Atomic Collisions and (e,2e) Reactions, Ed. P.J.O. Teubner and E. Weigold, IOP Conf. Series 122 (1992), pp. 171-180

[42] Neudatchin V.G., Smirnov Yu. F., Pavlitchenkov A.V. and Levin V. C. , Phys. Lett. 64A (1977), 31-4

[43] Smirnov Yu. F., Pavlitchenkov A.V. , Levin V. C. and Neudatchin V.G., J. Phys. B11,(1978), 3587

[44] Levin V. C. , Neudatchin V.G., Pavlitchenkov A.V. , and Smirnov Yu. F., J. Phys. B17,(1984), 1525

[45] Holland D.M.P., Codling K., West J.B. and Marr G.V. , J. Phys. B12 (1979), 2465 
[46] Avaldi L., Camilloni R., and Stefani G., "Correlation, wavefunctions and fundamental processes in gas phase photoionization : a proposal for experiments with synchrotron radiation ", (Roma 1987),IMAI-CNR RI/1/87

[47] Diviacco B., Bracco R., Poloni C., Walker R. P. and Zangrando D., Rev. Sci. Instrum. 63 (1992), 388

[48] Hoyer E., Chin J., Halbach K., Hassenzabl W.V., Humphries D., Kincaid B., Lancaster H. and Plate D., Rev. Sci. Instrum. 63 (1992), 359

[49] Flexman J.H., Hayes P.A., Smith P. and J.F. Williams in Book of Abstracts of Contributed Papers, XVII ICPEAC, McCarthy I.E., MacGillivray W.R. and Standage M.C. Ed. (Brisbane 1991), pp. 648

[50] Appell J., Durup J.,Fehsenfeld F.C., and Fournier P., J. Phys. B6 (1973),197

[51] Moddeman W.E., Carlson T.A., Krause M.O., Bullen B.P., Bull W.E., and Schweitzer G.K., J. Chem. Phys. 55 (1971), 2317

[52] Kim Y.B., Stephan K., Mark E., and Mark T.D. ,J. Chem. Phys. 74 (1981) , 6771

[53] Dorman F.H., and Morrison J.D., J. Chem. Phys. 35 (1961), 575

[54] Newton A.S. and Sciamanna A.F., J. Chem. Phys. 50 (1969), 4868

[55] Besnard M.], Hellner L., Malinovich Y., and Dujardin G., J. Chem. Phys. 85 (1986), 1316

[56] Fournier P.G. and March R.E., Chem. Phys. Lett. 137 (1987), 377

[57] Moore J.H., Tossell J.A., Coplan M.A., Cooper J.W. and Doering J.P., "Knockout reactions to study atomic and molecular electronic structure : the future. " in "(e,2e) and related processes", C.T. Whelan Ed. (Kluwer : Cambridge 1993) to be published

[58] Workshop on "Experimental aspects of multidetection and multicoincidence techniques", (Roma 1993), IMAI-CNR RI/4/92 